\title{
Association of Helicobacter pylori and Crohn's Disease Incidence: An Inversion Reaction?
}

\author{
Lars Erik Bartels ${ }^{1} \cdot$ Jens Frederik Dahlerup ${ }^{1}$
}

Received: 24 March 2017/Accepted: 28 March 2017/Published online: 5 April 2017

(C) Springer Science+Business Media New York 2017

Crohn's disease is a chronic inflammatory bowel disease assumed to be caused by genetic predisposition and environmental factors. In the past decades, both prevalence and incidence have increased in western countries [1]. Whereas the genetic makeup of populations is relatively stable over time, environmental influences are subject to short-term changes. Hence, the causes underlying an increase in Crohn's disease incidence and prevalence are most likely due to environmental changes.

A possible environmental factor is the spiral-shaped rugged bacterium Helicobacter pylori (H. pylori) which has been embedded in the human gastric mucosa for thousands of years [2]. Thriving in overcrowded societies with modest sanitary conditions, the bacterium has had splendid opportunities to infest entire populations in many past and present-day societies [3]. Being a well-known cause of ulcer and gastric cancer, it is perhaps surprising that $10-90 \%$ of the population worldwide carries this little troublemaker, often without any symptoms or overt disease.

Since improved living conditions and sanitation accompany economic growth, $H$. pylori infection rates have dropped substantially as societies become westernized [3]. Paradoxically, the decline of the $H$. pylori infection rate has been accompanied by an increased incidence of chronic inflammatory conditions such as Crohn's disease for no apparent reason [1], fueling speculation regarding a possible host-protective effect of $H$. pylori infection.

Lars Erik Bartels

larsbart@rm.dk

1 Department of Hepatology and Gastroenterology, Aarhus University Hospital, Nørrebrogade 44, 8000 Aarhus C, Denmark
In the current issue of Digestive Diseases and Sciences, an interesting article by Shah et al. [4] addresses this topic. In the article entitled "Is there a link between H. pylori and the epidemiology of Crohn's disease," the authors studied associations between $H$. pylori and Crohn's disease at an ecological level.

Several case-control and cohort studies have explored this subject since the first observations of Crohn's disease patients having lower frequency of $H$. pylori infections [5]. In several geographical settings, these studies all concluded that H. pylori and Crohn's disease are inversely correlated (see reference list in [4]).

Since this is only a correlation, several authors have sought possible explanation for this observation. For example, if Crohn's disease patients received antibiotics prior to H. pylori test as part of Crohn's or other therapy, eradicating $H$. pylori, the rate of $H$. pylori infections in Crohn's disease patients would be reduced. Perhaps $H$. pylori is only a proxy marker for exposure to an environment which protects against Crohn's disease, or is it a case of selection biases where patients due to regular doctor contact are tested for $H$. pylori more frequently? Other possible biases and confounders include exposure of Crohn's patients to other medical treatments that affect $H$. pylori, or just simply publication biases that skew the data. Of course, any biased conclusions would affect metaanalyses such as the ones listed in [4].

To meet the challenge of confounders and biases, Shah et al. took a novel approach by conducting an ecological epidemiologic study. Information about Crohn's disease prevalence and incidence was combined with geographical and time-matched H. pylori prevalence studies, with 22 incidence and 19 prevalence data pairs for Crohn's disease and $H$. pylori derived from 13 different countries. In that way, they were able to demonstrate yet again a robust 
Table 1 The key concepts of the editorial

What is the current knowledge?
Crohn's disease incidence and prevalence is increasing for unknown reasons
Although Helicobacter pylori infection is common, its prevalence is decreasing
Helicobacter pylori infection may affect the development of inflammatory diseases
What is new here?
In an ecological study, Helicobacter pylori and Crohn's disease are inversely correlated
Crohn's disease development may be affected by Helicobacter pylori infection
Where to go from here?
The inverse epidemiological association is evident. Further epidemiological studies seem pointless
Attention should be directed to possible mechanistic effects of Helicobacter pylori beneficial to the development of Crohn's disease

inverse correlation between H. pylori infection and Crohn's disease prevalence and incidence. The higher the proportion of the population infected with $H$. pylori, the lower the risk of Crohn's disease. The authors argue that their study approach functions as a confounder control as it focuses on a regional versus an individual level.

Shah et al. present a thorough examination of the literature on Crohn's disease epidemiology from 1990 to 2016 combining these data with results on $H$. pylori infection prevalence. Nevertheless, the study still has several potential pitfalls. First of all, exposure to $H$. pylori may not be the only characteristic that distinguishes the exposed group from the non-exposed groups. Still, the use of antibiotics could have been distributed unevenly and affected results. Secondly, Shah et al. retrieved their data from a prolonged period (1990-2016). From the knowledge gained from previous studies, it is very likely that $H$. pylori infection rate had been reduced [6], while Crohn's disease incidence and prevalence increased in the studied areas over that time period, potentially biasing the expectations of the investigators. Thirdly, it would have been very interesting if authors had included developing countries below the 20,000 USD per capita gross domestic product threshold. These countries may have experienced the largest change in H. pylori prevalence and Crohn's disease incidence and prevalence in the period 1990-2016. They could have given additional information although data regarding Crohn's disease incidence and prevalence could be erroneously low due to lack of diagnosis in developing healthcare systems.

Setting aside all reservations, the present study is an important contribution that supports the notion that the incidences of $H$. pylori and Crohn's disease are inversely correlated. As studies with this consistent conclusion are becoming more and more frequently published, the robust inverse correlation between $H$. pylori and Crohn's disease seems evident. The big question is why are they inversely correlated? In the present study, the authors speculate that
"H. pylori may have direct immunomodulatory effects." Although mechanistic studies are few and lack convincing causal explanations, it has been suggested that $H$. pylori induces the development of regulatory $\mathrm{T}$ cells and impairs dendritic cell maturation, with a consequent tolerogenic phenotype [7] as part of the mechanisms used by $H$. pylori to avoid host immune reactions that inadvertently fail to inhibit the development of inflammatory conditions like Crohn's disease.

Nevertheless, we have performed a study that indicates that the mechanisms underlying the correlation may be more complex since factors other than $H$. pylori infection alter the development to Crohn's disease [8]. In a general practice cohort tested for $H$. pylori by the urea breath test, we studied Crohn's disease prevalence at the time of the test and incidence in a 6-year follow-up. At the time of test, H. pylori infection was associated with lower Crohn's disease prevalence, a correlation that held after probable $H$. pylori eradication, wherein $H$. pylori infection prior to testing was associated with a lower Crohn's disease incidence. One interpretation of these results is that any disease-modifying effects of $H$. pylori infection occurred earlier in life-for example in childhood-supported by the observation that $H$. pylori seems to protect against Crohn's disease even in children. Alternatively, it could mean that $H$. pylori is merely an indicator of a lifestyle protective against Crohn's disease rather than a causal factor.

As suggested by Shah et al., H. pylori may "be a marker for....other gastrointestinal infections" that could protect against Crohn's disease. Interestingly in this context, the microbiota in the upper gastrointestinal tract differ between $H$. pylori positive and negative individuals, possibly due to the observation that $H$. pylori infection increases gastric $\mathrm{pH}$ [9]. Another recent study reported that the microbiota also changed after eradication of $H$. pylori [10]. As the gastrointestinal microbiota is believed to substantially contribute to Crohn's disease development, a change in the 
composition of the microbiota may change the susceptibility to Crohn's disease.

Reviewing Shah et al.'s study and prior epidemiologic studies, conducting further studies focusing on the association between Crohn's disease and $H$. pylori seems pointless. Since the association is evident, future studies should rather draw attention to mechanistic factors of $H$. pylori infection, in particular during childhood. Alternatively, studies should focus on concurrent factors to $H$. pylori infection, for example changes in the gastrointestinal microbiota that may also affect the pathogenesis of Crohn's disease. Data obtained from studies of this nature should ultimately inform the decision regarding the eradication of H. pylori in asymptomatic individuals - in particular in patients with established Crohn's disease (Table 1).

\section{Compliance with ethical standards}

Conflict of interest None.

\section{References}

1. Ananthakrishnan AN. Epidemiology and risk factors for IBD. Nat Rev Gastroenterol Hepatol. 2015;12:205-217. doi:10.1038/ nrgastro.2015.34.
2. Megraud F, Lehours P, Vale FF. The history of Helicobacter pylori: from phylogeography to paleomicrobiology. Clin Microbiol Infect. 2016;22:922-927.

3. Calvet X, Ramirez Lazaro MJ, Lehours P, Megraud F. Diagnosis and epidemiology of Helicobacter pylori infection. Helicobacter. 2013;18:5-11. doi:10.1111/hel.12071.

4. Shah A, Talley N, Walker M, et al. Is there a link between $H$. pylori and the epidemiology of Crohn's disease? Dig Dis Sci. (Epub ahead of print). doi:10.1007/s10620-017-4496-z.

5. el-Omar E, Penman I, Cruikshank G, et al. Low prevalence of Helicobacter pylori in inflammatory bowel disease: association with sulphasalazine. Gut. 1994;35:1385-1388.

6. Dahlerup S, Andersen RC, Nielsen BS, et al. First-time urea breath tests performed at home by 36,629 patients: a study of Helicobacter pylori prevalence in primary care. Helicobacter. 2011;16:468-474. doi:10.1111/j.1523-5378.2011.00872.x.

7. Arnold IC, Hitzler I, Muller A. The immunomodulatory properties of Helicobacter pylori confer protection against allergic and chronic inflammatory disorders. Front Cell Infect Microbiol. 2012;2:10. doi:10.3389/fcimb.2012.00010.

8. Bartels LE, Jepsen P, Christensen LA, Gerdes LU, Vilstrup H, Dahlerup JF. Diagnosis of Helicobacter pylori infection is associated with lower prevalence and subsequent incidence of Crohn's disease. J Crohns Colitis. 2016;10:443-448.

9. He C, Yang Z, Lu N. Imbalance of gastrointestinal microbiota in the pathogenesis of Helicobacter pylori-associated diseases. Helicobacter. 2016;21:337-348. doi:10.1111/hel.12297.

10. Yap TW, Gan HM, Lee YP, et al. Helicobacter pylori eradication causes perturbation of the human gut microbiome in young adults. PLoS ONE. 2016;11:e0151893. doi:10.1371/journal.pone. 0151893 . 